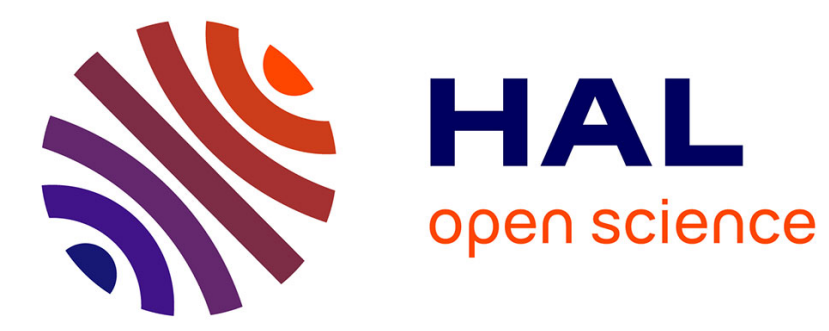

\title{
Cristallogénèse du rubis par zone flottante dans un four à résistance
}

\author{
G. Petit-Le Du, J. Aubrée
}

\section{To cite this version:}

G. Petit-Le Du, J. Aubrée. Cristallogénèse du rubis par zone flottante dans un four à résistance. Revue de Physique Appliquée, 1967, 2 (4), pp.264-270. 10.1051/rphysap:0196700204026400 . jpa-00242803

\section{HAL Id: jpa-00242803 https://hal.science/jpa-00242803}

Submitted on 1 Jan 1967

HAL is a multi-disciplinary open access archive for the deposit and dissemination of scientific research documents, whether they are published or not. The documents may come from teaching and research institutions in France or abroad, or from public or private research centers.
L'archive ouverte pluridisciplinaire HAL, est destinée au dépôt et à la diffusion de documents scientifiques de niveau recherche, publiés ou non, émanant des établissements d'enseignement et de recherche français ou étrangers, des laboratoires publics ou privés. 


\title{
GRISTALLOGÉNÈSE DU RUBIS PAR ZONE FLOTTANTE DANS UN FOUR A RÉSISTANGE
}

\author{
Par G. PETIT-LE DU et J. AUBRÉE, \\ Département Physique-Chimie-Métallurgie du Centre National d'Études des Télécommunications, 92-Issy-les-Moulineaux.
}

\begin{abstract}
Résumé. - On décrit un appareillage et un procédé de fabrication de monocristaux de rubis, utilisant la technique de la zone flottante par chauffage à résistance sous atmosphère d'argon.

Des monocristaux de forme cylindrique $6 \times 60 \mathrm{~mm}$, avec une concentration en $\mathrm{Cr}^{3+}$ de $500 \mathrm{ppm}$, d'orientation [0001], ont été obtenus. La vitesse de croissance est de $14 \mathrm{~mm} / \mathrm{h}$. Les densités moyennes de dislocations sont inférieures à $10^{4} \mathrm{~cm}^{-2}$. L'effet laser a été observé sur les cristaux réalisés par cette méthode.

Abstract. - Apparatus and process for growing ruby single-crystals, using the floating zone technique by resistance heating under an argon atmosphere, are described.

Cylindrical $6 \times 60 \mathrm{~mm}$ single-crystals, with a $500 \mathrm{ppm} \mathrm{Cr}^{3+}$ concentration, [0001]-axis orientation, were obtained. Growth rate was $14 \mathrm{~mm} / \mathrm{h}$. Average dislocation densities were less than $10^{4} \mathrm{~cm}^{-2}$. The laser effect was observed on the crystals grown by this method.
\end{abstract}

I. Introduction. - Dans le cadre de l'étude des techniques de préparation des matériaux pour lasers à solides fluorescents, nous avons conçu et expérimenté un procédé de croissance rapide du rubis [1].

Les principales techniques de fabrication de corindon et rubis monocristallins sont les suivantes :

1. LA MÉthode DE Verneuil [2] AVEG FUSION DE POUDRE $\mathrm{Al}_{2} \mathrm{O}_{3}+\mathrm{Cr}_{2} \mathrm{O}_{3}$ AU GHALUMEAU OXHYDRIQUe. - Universellement utilisée depuis plus d'un demi-siècle pour les besoins de la joaillerie et de l'horlogerie, cette technique, appliquée de manière assez rudimentaire jusqu'à ces dernières années, a été étudiée avec un intérêt nouveau et s'est vue sensiblement modifiée et perfectionnée [3] lorsque le laser a nécessité des cristaux de qualité améliorée; plusieurs modes de chauffage ont été proposés en remplacement du chalumeau oxhydrique, trop brutal et difficile à stabiliser pour une longue durée : four à image [4], torche à plasma [5], sans qu'il soit possible néanmoins d'éviter ou d'éliminer ultérieurement de façon satisfaisante les tensions d'origine thermique et les désorientations réticulaires qui caractérisent généralement les rubis élaborés par ce procédé; de plus, ces derniers ne peuvent en pratique croître que dans une seule direction cristallographique : celle où l'axe optique est à environ $60^{\circ}$ de l'axe géométrique du cristal.

2. LA REGRISTALLISATION A PARTIR D'UN SOLVANT [6] ET AUTRES APPLICATIONS DU MÊME PHÉNOMÈNE [7], [8]. - Ce procédé est long, et a produit difficilement jusqu'à présent des cristaux homogènes d'assez grandes dimensions et exempts de défauts ou d'inclusions.

3. LA SYNTHÈSE HYDROTHERMALE [9], [10]. - On retrouve ici les mêmes inconvénients que dans le cas précédent.

4. Le tirage de Czochralski [11], [12]. - Cette méthode a connu récemment un grand essor dans l'industrie des semi-conducteurs, et il était évident qu'on chercherait à l'appliquer au corindon et au rubis, bien qu'on se soit heurté à de sérieuses complications technologiques en raison du point de fusion élevé de l'alumine; la technique originale de la fusion en autocreuset [13] semblerait offrir ici un certain intérêt, bien que sa mise au point doive soulever quelque difficulté; le tirage a déjà permis de produire des rubis de grandes dimensions et contenant peu de dislocations; il faut remarquer que, de même que dans le cas du procédé Verneuil, la seule direction de tirage pratiquement utilisable se situe à $60^{\circ}$ de l'axe optique.

5. LA zone flottante [14]. - Le chauffage étant réalisé par une résistance métallique plate servant également de support à la zone fondue, les cristaux fabriqués de cette manière sont de qualité assez médiocre; on pourrait aussi envisager la monocristallisation de l'alumine par fusion de zone dans un four à image [15]. 
6. La Groissance en phase vapeur [16]. - Le processus de formation est relativement lent, mais les cristaux obtenus présentent des densités de dislocations de l'ordre de $10^{2} \mathrm{~cm}^{-2}$, ce qui constitue une valeur minimale, difficile à atteindre par les autres méthodes.

7. LA REGRISTALLISATION EN PHASE SOLIDE [17]. La mise en ouvre de cette technique, qui semble permettre la réalisation de cristaux de dimensions appréciables et de bonne qualité, fait appel à des températures et pressions difficiles à concilier.

Compte tenu des avantages et inconvénients présentés par les diverses méthodes connues lors du démarrage de notre étude, nous avons mis au point un four de cristallogenèse du rubis permettant :

- La croissance rapide de monocristaux de forme cylindrique, de dimensions $6 \times 60 \mathrm{~mm}$ et d'orientation cristallographique quelconque;

- Le refroidissement contrôlé et, éventuellement, le recuit in situ des cristaux ainsi obtenus, ce qui a pour effet de les libérer de toute tension d'origine thermique.

La technique conçue initialement s'inspirait du principe et de la disposition de celle de Verneuil, à cela près que le chauffage de la couche fondue à la surface du cristal, disposé verticalement, était assuré par une résistance de tungstène et que la croissance était alimentée par la chute à intervalles réguliers de sphérules de rubis préalablement chauffées dans un distributeur adéquat [1]. Le déroulement de l'étude nous a conduits à abandonner ce mode d'alimentation, sur lequel nous ne nous étendrons pas dans cet article, pour le remplacer par une alimentation au moyen d'une baguette cylindrique, celle-ci étant, de même que les sphérules, taillée dans une boule Verneuil ordinaire. Du fait de cette modification, la physionomie actuelle de l'ensemble se rapproche plus de la zone flottante que de la méthode Verneuil.

II. Appareillage. - Le four est contenu dans une enceinte à vide cylindrique d'axe vertical (fig. 1) fermée à ses extrémités par des platines, la platine supérieure mobile permettant un accès facile, et reliée par un orifice de grand diamètre à un dispositif de pompage comprenant une pompe à palettes et une pompe à diffusion d'huile. Cette enceinte et ses platines terminales sont à double paroi avec circulation d'eau entre les deux parois. Elle peut être balayée par un courant gazeux grâce à deux ajutages appropriés. L'ensemble a été construit par La Physique Appliquée. La description qui va suivre sera mieux comprise à l'aide de la figure 2.

Dans l'axe de l'enceinte à vide, un germe monocristallin de rubis 11 est centré dans un logement cylindrique qui constitue l'extrémité supérieure d'une

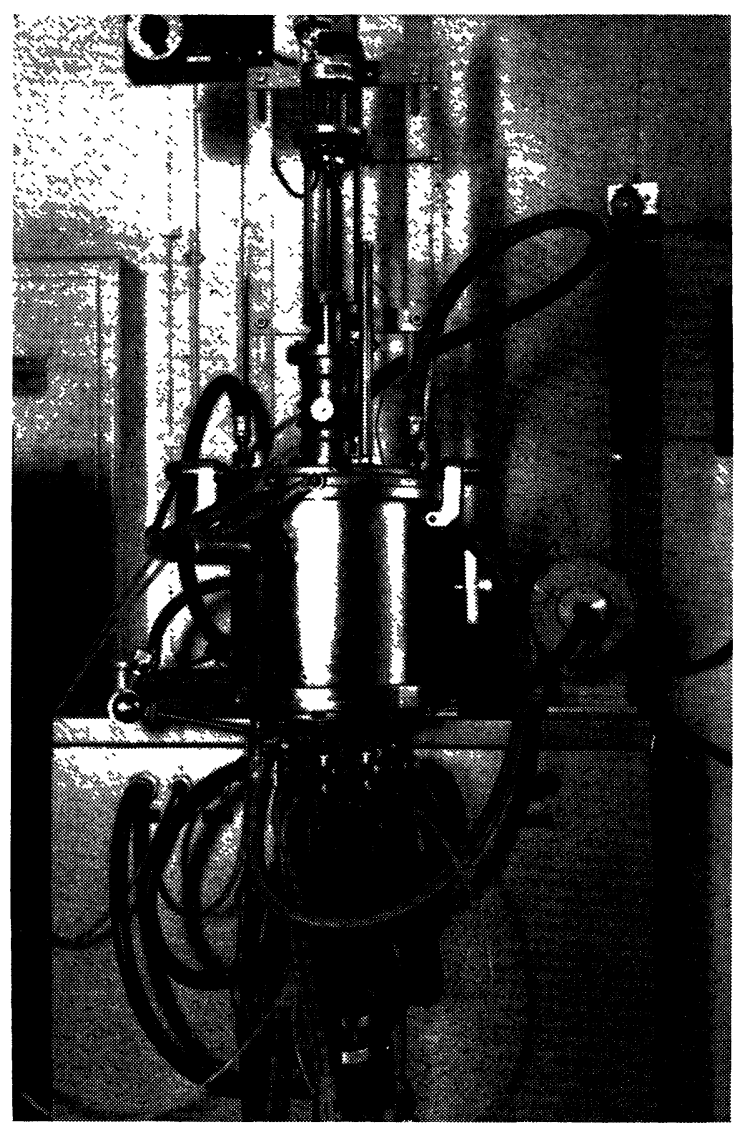

FIG. 1. - Vue extérieure de l'installation.

tige de molybdène 14 verticale, dont l'extrémité inférieure vient s'adapter sur une tige d'acier inoxydable, refroidie intérieurement par une circulation d'eau. Cette tige sort de l'enceinte à travers un joint étanche pour être en relation avec un ensemble mécanique actionné par des moteurs assurant la rotation et la descente du cristal. Un dispositif identique, à la partie supérieure de l'enceinte, communique au support 1 de la baguette d'alimentation un double mouvement semblable à celui du germe. La double paroi de l'enceinte est percée d'un hublot fermé par une plaque de verre de silice et sur lequel peut être fixée une lunette grossissante permettant d'observer l'extrémité du cristal, son alimentation et sa croissance.

Dans la partie centrale de l'enceinte et coaxialement à celle-ci sont disposées les résistances 9 et 13 séparées l'une de l'autre par un écran 6 B qui établit la limite entre la chambre supérieure de fusion et la chambre inférieure de refroidissement contrôlé. La résistance 9 est constituée par un anneau à section rectangulaire ( fig. 4) usiné dans un barreau de tungstène massif, un espace suffisant étant ménagé entre les deux amenées de courant pour permettre l'observation du cristal au moyen de la lunette sus-mentionnée. Ces deux ame- 


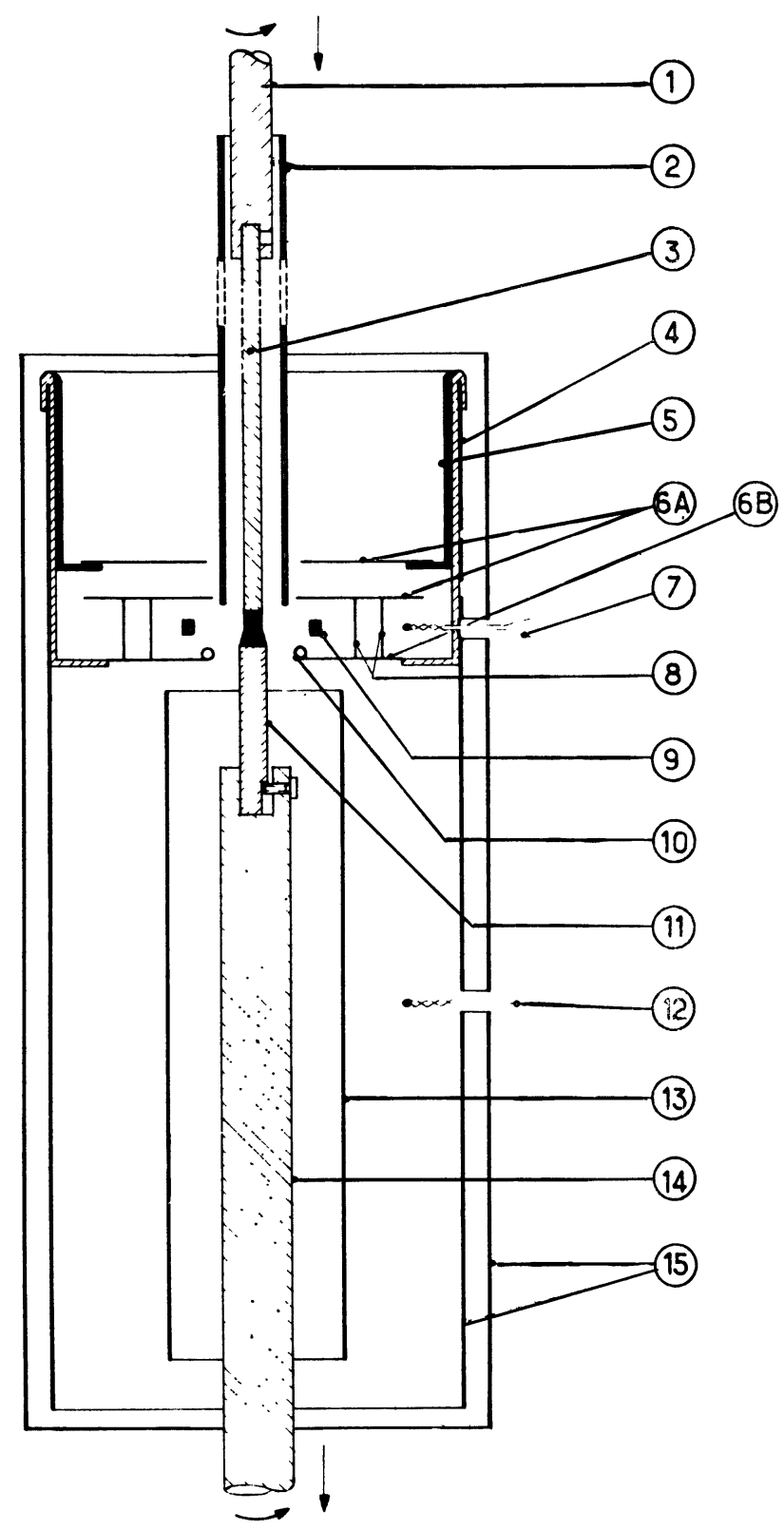

FIG. 2. - Schéma des chambres de fusion et de refroidissement contrôlé : 1. Support baguette de rubis (Mo). - 2. Pièce protectrice de la baguette (Ta). - 3. Baguette de rubis. - 4. Support écran (W). - 5. Support écran (W). - 6. Écrans (W). - 7. Thermocouple. 8. Écrans (W). - 9. Élément chauffant (W). 10. Jonc $(\mathrm{W})$. - 11. Germe de rubis. - 12. Thermocouple. - 13. Élément chauffant $(\mathrm{Ta})$. - 14. Support germe (Mo). - 15. Écrans (Ta).

FIG. 4.

Résistance en tungstène de la chambre de fusion.

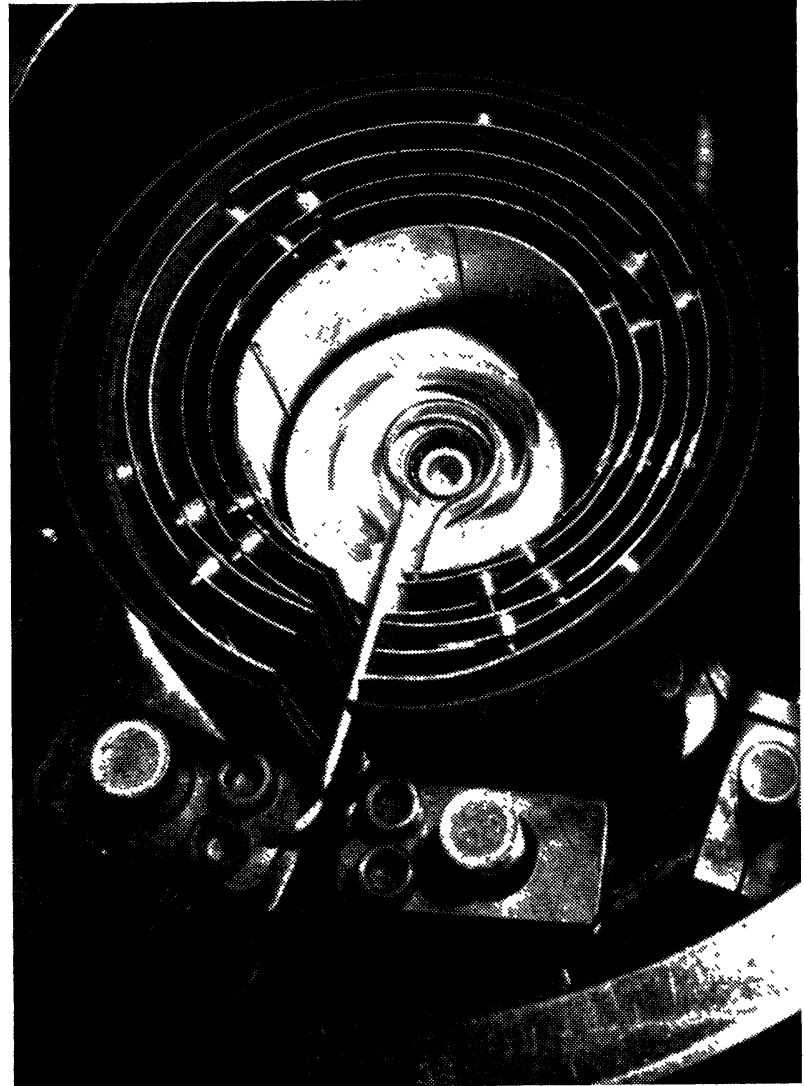

FIG. 3. - Vue intérieure de la chambre de fusion.

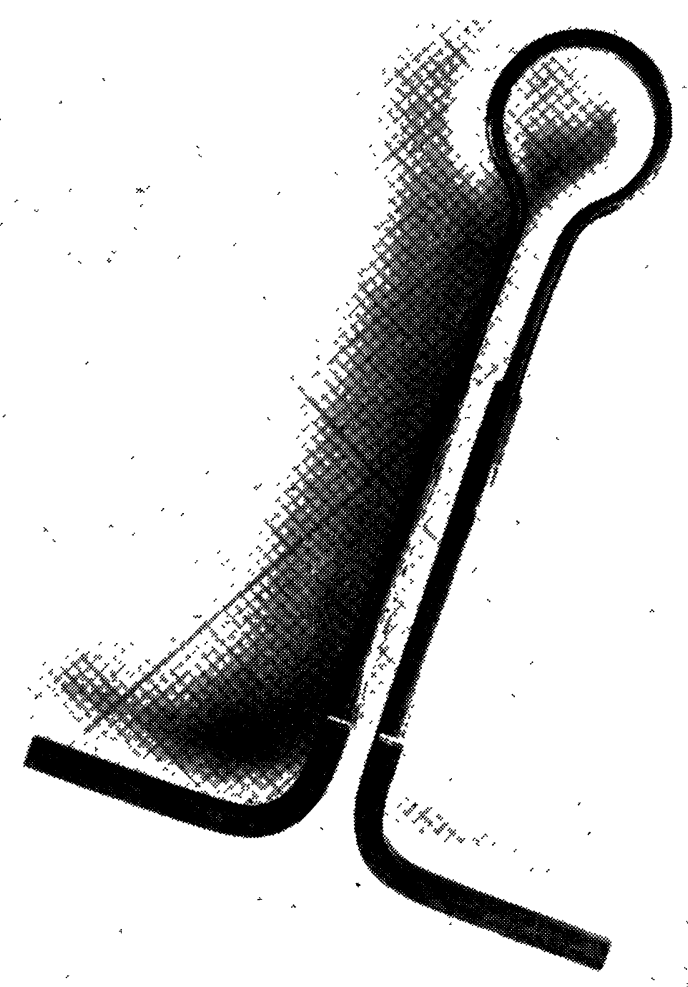


nées sont connectées à des conducteurs verticaux creux refroidis par circulation interne d'eau et fixés à la platine inférieure de l'enceinte. La résistance 13 est une feuille de tantale cylindrique découpée en créneaux; elle est disposée coaxialement à la résistance 9 , en dessous de l'écran $6 \mathrm{~B}$, et alimentée par des connexions radiales aboutissant à des conducteurs verticaux du même type que les précédents. Durant la croissance d'un cristal, les intensités admises dans les résistances supérieure et inférieure sont respectivement de $300 \mathrm{~A}$ sous $8,6 \mathrm{~V}$ et de $130 \mathrm{~A}$ sous $4 \mathrm{~V}$.

Afin de limiter les pertes de chaleur par rayonnement, un jeu de sept écrans cylindriques entoure l'ensemble des deux chambres. Ces écrans sont concentriques, les plus extérieurs étant en inconel, puis en molybdène, et les deux intérieurs en tantale. Dans ces écrans ont été pratiquées les échancrures nécessaires pour dégager le champ d'observation du hublot et le passage des connexions des résistances. Chacun des écrans verticaux est complété par deux écrans horizontaux disposés à ses extrémités, les écrans supérieurs étant fixés à la platine supérieure par des entretoises. Tous les écrans horizontaux sont percés en leur centre d'un trou circulaire permettant le passage des supports du germe et de la baguette d'alimentation. La chambre de fusion comporte deux écrans supplémentaires 6 A et 8 en tungstène, horizontaux et verticaux. Autour de la baguette d'alimentation 3 est disposé un tube de tantale 2, centré sur la platine supérieure de l'enceinte, et qui descend jusque dans la chambre de fusion : de la position de ce tube de protection dépend l'emplacement de la limite supérieure de la zone fondue.

La mesure des températures des chambres de fusion et de refroidissement contrôlé est effectuée au moyen de deux thermocouples 7 et 12 en tungstène $-5 \%$ rhénium/tungstène $-26 \%$ rhénium placés au voisinage des résistances. Il faut préciser que ces thermocouples ne commandent, en l'état actuel de notre installation, aucune régulation de température : leurs indications fournissent des points de repère à l'opérateur qui établit son programme de chauffage d'après le contrôle visuel de la croissance du cristal et l'applique en manœuvrant des alternostats alimentés sur secteur stabilisé. En l'absence de régulation-programmation de température, une très grande habileté du manipulateur est indispensable.

III. Mode opératoire. - Deux remarques préliminaires :

- Avant chaque opération, il est nécessaire de vérifier le parfait centrage de tous les éléments du four : résistances, écrans, supports du germe et de la baguette, tube de protection de la baguette;

- La mise en place d'une résistance neuve demande un dégazage à $2000^{\circ} \mathrm{C}$, sous vide d'environ $10^{-5}$ torr, durant $2 \mathrm{~h}$.
Les différentes phases de la fabrication d'un rubis vont maintenant être décrites.

Le germe cylindrique, préalablement orienté aux rayons $\mathrm{X}$, est rodé en calotte hémisphérique à sa partie supérieure, de manière à faciliter la formation du ménisque au moment de la fusion superficielle. Il est ensuite placé sur son support, dans lequel il est maintenu sans jeu par un ergot de molybdène le serrant sur un méplat. La baguette d'alimentation est rodée et taillée, puis fixée sur son support de la même manière que le germe. Ajoutons que germe et baguette sont soigneusement nettoyés avant emploi : dégraissage, suivi d'un décapage chimique dans l'acide orthophosphorique à $200^{\circ} \mathrm{C}$ pendant $2 \mathrm{mn}$.

L'enceinte est fermée. On effectue un dégazage à $1600^{\circ} \mathrm{C}$ sous vide de $10^{-5}$ torr durant $30 \mathrm{mn}$. On laisse refroidir, puis on casse le vide en introduisant de l'argon $\mathrm{U}$, séché sur tamis moléculaires régénérés avant chaque utilisation : l'absence d'humidité, de même que celle d'oxygène, est importante pour le maintien en bon état des éléments du four, facilement oxydables à haute température. Un balayage d'argon de $2 \mathrm{l} / \mathrm{mn}$, à une pression légèrement supérieure à la pression atmosphérique et réglée par une soupape, subsiste jusqu'à la fin de l'opération.

Le germe et la baguette, animés d'un mouvement de rotation dans le même sens de 10 et $30 \mathrm{tr} / \mathrm{mn}$ respectivement, sont amenés presque au contact l'un de l'autre à l'intérieur de la chambre de fusion, tandis que les deux résistances sont mises sous tension. Lorsque la chambre inférieure atteint la température de $1950^{\circ} \mathrm{C}$, cette dernière est stabilisée, après quoi le chauffage de la chambre supérieure est progressivement augmenté jusqu'à fusion superficielle des deux pièces de rubis. La température de la chambre supérieure est alors élevée de $40^{\circ} \mathrm{C}$; simultanément, la baguette est descendue jusqu'à établissement d'une zone fondue continue entre germe et baguette. Cette phase du démarrage est très critique : un chauffage trop fort risque de provoquer une coulée du liquide, suivie d'une rupture de la zone; par contre, un chauffage insuffisant peut amener la mise en contact des deux interfaces solide-liquide, fortement convexes l'un vers l'autre au centre de la masse liquide (fig. 5), d'où la formation de défauts au cœur du cristal.

Les moteurs de descente sont mis en route après une période de stabilisation de quelques minutes. Le mouvement du cristal se fait à la vitesse de $14 \mathrm{~mm} / \mathrm{h}$. La vitesse de descente de la baguette est fonction du rapport des diamètres du germe et de la baguette. Pour des diamètres respectifs de $6 \mathrm{~mm}$ et $5 \mathrm{~mm}$, le germe conserve une section constante pour une vitesse de déplacement de la baguette de $17,5 \mathrm{~mm} / \mathrm{h}$. Durant toute la croissance, la température de la chambre de fusion est élevée régulièrement, l'augmentation étant de $25^{\circ} \mathrm{C}$ pour une longueur de cristal de $60 \mathrm{~mm}$. 


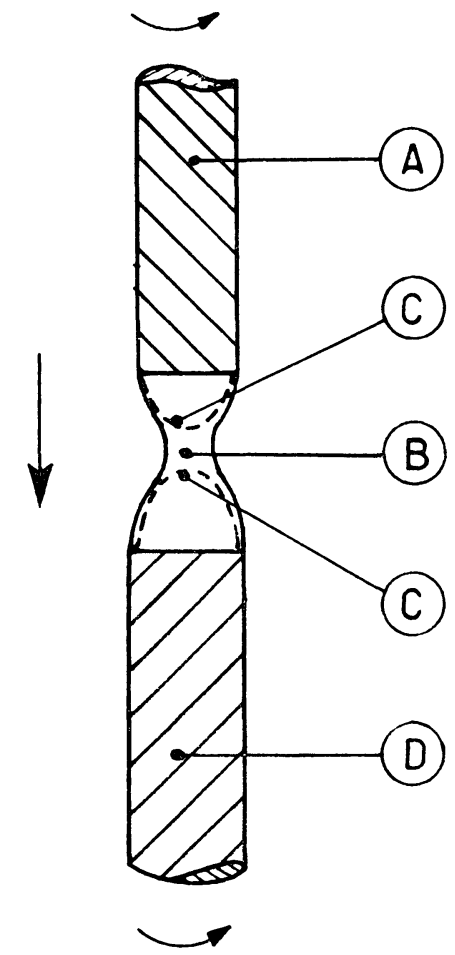

FIG. 5. - Morphologie de la zone fondue : A, baguette d'alimentation. - B, zone fondue. - C, interface solide-liquide. - $\mathrm{D}$, germe.

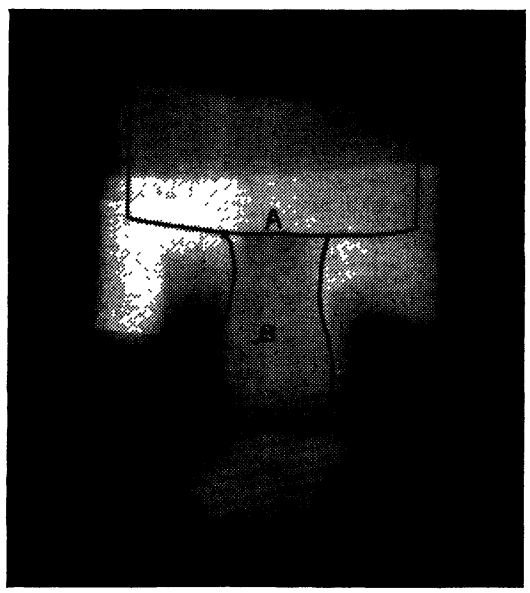

FIG. 6. - Cristal de rubis en cours de croissance. $A$ : pièce de protection numérotée 2 sur la figure 2 . $\mathrm{B}$ : zone fondue. $-\mathrm{C}$ : extrémité du germe. - D : écran numéroté $6 \mathrm{~B}$ sur la figure 2 (la limite supérieure de la zone fondue se situe à l'intérieur de la pièce $\mathrm{A}$ ).

Pour arrêter l'opération, il suffit de ramener la température de la chambre de fusion à sa valeur initiale tout en remontant rapidement la baguette jusqu'à rupture de la zone liquide. L'abaissement de température évite le risque d'une coulée terminale. Le refroidissement de l'ensemble se fait en $12 \mathrm{~h}$ environ.
IV. Résultats obtenus. - Le procédé décrit ci-dessus permet d'obtenir couramment des monocristaux ( fig. 7) de caractéristiques reproductibles. La direction de croissance utilisée jusqu'à présent correspond généralement à l'axe [0001].

La qualité cristalline, évaluée au test de Lambot (fig. 8), est supérieure à celle des rubis Verneuil ordi-

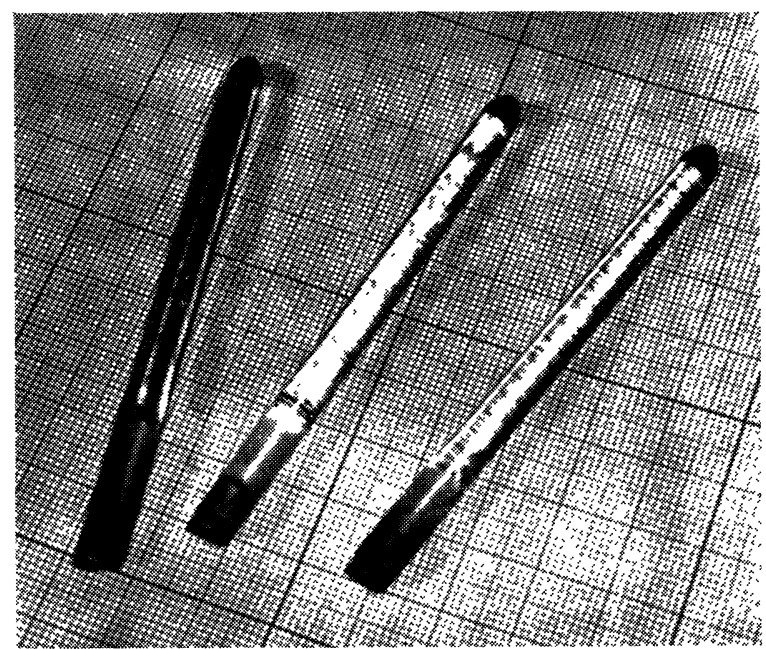

FIG. 7. - Monocristaux de rubis.

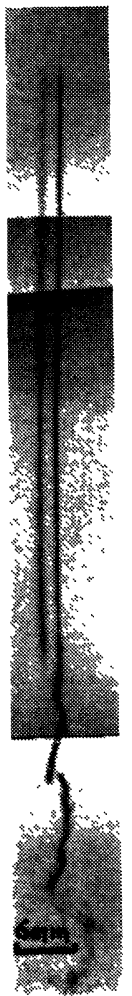

Fig. 8. - Diagramme de Lambot : $6 \mathrm{~mm}$ sur l'échelle horizontale correspondent à une désorientation de $1^{\circ}$. On remarque la différence de qualité entre le germe (extrémité inférieure du diagramme) et le cristal. 
naires. (Rappelons que le test de Lambot consiste à mesurer la déformation subie par un faisceau convergent de rayons $\mathrm{X}$ monochromatiques arrivant sur le cristal suivant une incidence de Bragg, cette déformation étant fonction des désorientations existant à l'intérieur de la famille de plans réticulaires sur lesquels se produit la réflexion.) On note, dans certains cas, la présence de microdéfauts dont l'emplacement correspond à un abaissement accidentel de la température dans la chambre de fusion.

La contamination en tungstène et tantale due aux résistances et écrans est de l'ordre de $1 \mathrm{ppm}$ en fin de cristal. La concentration moyenne en chrome est de $500 \mathrm{ppm}\left(\mathrm{Cr}_{2} \mathrm{O}_{3} / \mathrm{Al}_{2} \mathrm{O}_{3}\right.$ en poids $)$ à partir de baguettes dopées à $1000 \mathrm{ppm}$. La perte en chrome est donc de $50 \%$ environ. Ce résultat est à comparer à celui obtenu dans le cas des rubis Verneuil [18], où la perte en chrome atteint $70 \%$. La répartition longitudinale du chrome est satisfaisante; par contre, on remarque dans le sens radial une inhomogénéité systématique (fig. 9) dont l'origine n'a pu encore être décelée.

Les densités de dislocations, révélées par la méthode

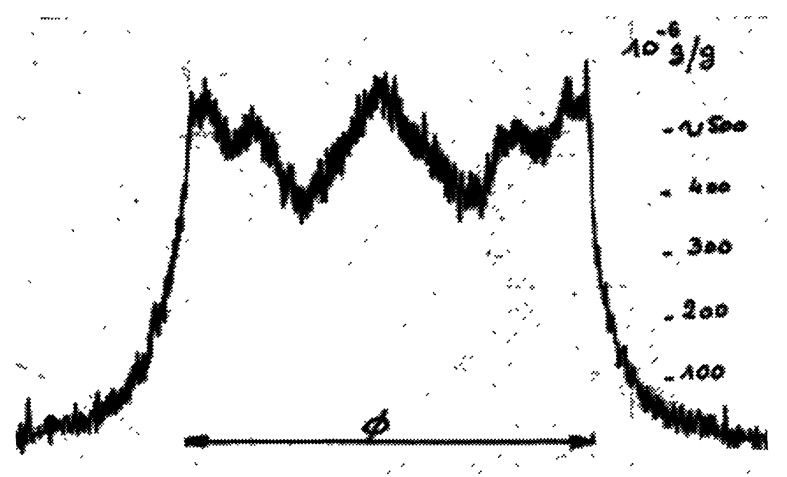

FIG. 9. - Répartition radiale du chrome.

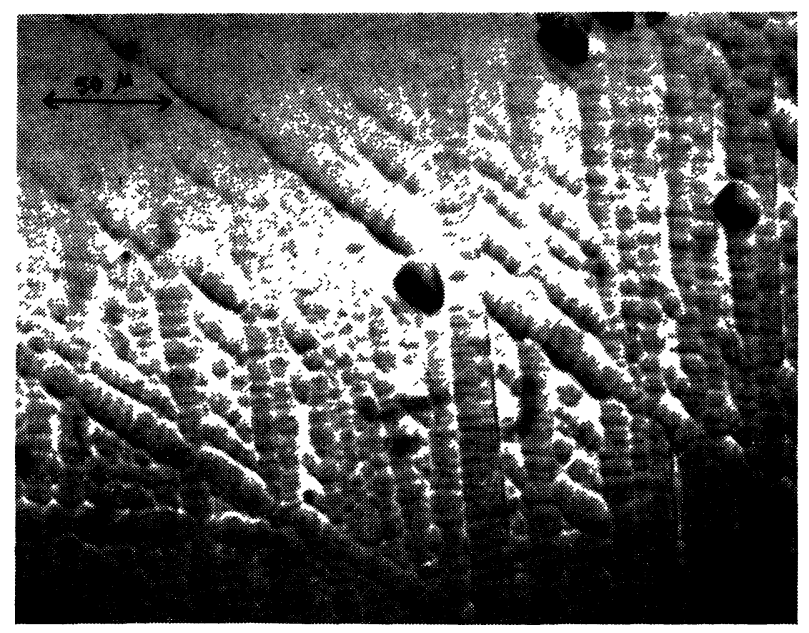

FIG. 10. - Dislocations. $3000 \mathrm{~cm}^{-2}$, plan [0001]. de Alford et Stephens [19], sont faibles et atteignent des valeurs moyennes inférieures à $10^{4} \mathrm{~cm}^{-2}$ ( fig. 10).

Des mesures de l'effet laser ont été effectuées sur certains des premiers cristaux réalisés. On a pu mesurer un seuil d'excitation de $1000 \mathrm{~J}$ et une énergie de sortie de $64 \mathrm{~mJ}$ pour une excitation de $1500 \mathrm{~J}$. Des rubis Verneuil classiques étudiés dans les mêmes conditions sur le même banc de mesures présentaient, pour un seuil et une excitation du même ordre de grandeur, une énergie de sortie d'environ $10 \mathrm{~mJ}$. Le diamètre du faisceau est d'environ $1 \mathrm{~cm}$ à une distance de $2 \mathrm{~m}$. L'essai systématique en laser est actuellement en cours sur des séries de cristaux réalisés suivant diverses conditions de croissance.

V. Conclusion. - La méthode qui vient d'être présentée est capable de produire rapidement, avec un matériel relativement simple, des rubis de qualité cristalline égale ou supérieure à celle que peut permettre n'importe quelle autre méthode actuellement connue. La direction de croissance [0001], d'un emploi malaisé dans les techniques offrant par ailleurs la possibilité de réaliser des cristaux de bonne qualité, a été utilisée ici avec succès.

Les études actuellement poursuivies sur le rubis, en attendant l'application du procédé à d'autres matériaux, tendent à améliorer certaines caractéristiques des cristaux tout en simplifiant la méthode de fabrication.

L'amélioration des cristaux consistera essentiellement à éliminer la variation radiale de la répartition du chrome. Il semble que des essais portant sur les conditions de croissance (vitesses de rotation et de déplacement, pression de gaz dans l'enceinte) ou sur des cycles de recuit dans différents gaz permettront d'apporter une solution à ce problème. En ce qui concerne les dimensions des cylindres de rubis fabriqués, il serait facile d'en augmenter la hauteur par une modification de détail de l'appareillage; pour le diamètre, par contre, il est évident que le principe même de la méthode ainsi que le mode de chauffage de la zone flottante interdisent d'envisager une augmentation sensible, principalement dans le cas du rubis dont la densité est relativement forte, et faible la tension de surface.

La simplification du procédé de fabrication pourrait comporter, d'une part, l'installation d'un régulateurprogrammeur de température qui permettrait d'éviter la présence continuelle d'un opérateur expérimenté durant toute la croissance d'un cristal, d'autre part, le remplacement, comme matériau d'alimentation de la zone fondue, d'une baguette de rubis par une tige de poudres frittées $\mathrm{Al}_{2} \mathrm{O}_{3}+\mathrm{Cr}_{2} \mathrm{O}_{3}$. Ce remplacement supprimerait la sujétion du découpage et du tournage, toujours compliqués et onéreux dans le cas d'un corps aussi dur que le rubis.

Manuscrit reçu le 28 juin 1967. 


\title{
BIBLIOGRAPHIE
}

[1] Petiti-Le Du (G.), Aubrée (J.), Brevet français no 1.432.731 délivré le 14-2-1966.

[2] Verneuir, (A.), C. R. Acad. Sc., 1902, 135, 791.

[3] Reiss (F. A.), Crystal Growth : Proceed. I.C.C.G., Boston, juin 1966, 63.

[4] Halden (F. A.), SeDLACEK (R.), Rev. Scient. Instr., 1963, 34, 622 .

[5] REED (T. B.), J. Appl. Phys., 1961, 32, 2534.

[6] White (E. A. D.), Nature (G.B.), 1961, 191, 901.

[7] Linares (R. C.), J. Appl. Phys., 1964, 35, 433.

[8] Cerrclet (M.), Rapport interne CNET : Document no PCM 138, 1965.

[9] Laudise (R. A.), Ballman (A. A.), J. Amer. Chem. Soc., 1958, 80, 2655.

[10] Marais (M.), Rapport interne CNET : Document no PCM 133, 1965.

[11] Paladino (A. E.), Roiter (B. D.), J. Amer. Ceram. Soc., 1964, 47, 465.
[12] Charvat (F. R.), Smith (J. C.), Nestor (O. H.), Crystal Growth : Proceed. I.C.C.G., Boston, juin 1966, 45.

[13] PeReZ y Jorba (M.), Collongues (R.), Rev. Hautes Tempér. et Réfract., 1964, 1, 21.

[14] Cockayne (B.), Chessnas (M.), Gasson (D. B.), J. Mat. Sc., 1967, 1, 7.

[15] Kooy (C.), CouWENBERG (H. J. M.), Rev. Techn. Philips, 1962, 23, 155.

[16] Schaffer (P. S.), Comm. Symposium Ceramics Processed Subconventional Temperatures, Chicago, avril 1964.

[17] Selifers (D. J.), Heuer (A. H.), Rhodes (W. H.), VAsilos (T.), J. Am. Ceram. Soc., 1967, 50, 217.

[18] Sil'Nichenko (V. G.), Gritsenko (M. M.), Sov. Phys. Cristall., 1965, 9, 647.

[19] AlfoRd (W. J.), Stephens (D. L.), J. Am. Ceram. Soc., 1963, 46, 193.

\section{MESURE DES TEMPS DE RELAXATION SPIN-RÉSEAU DES IONS PARAMAGNÉTIQUES PAR UNE MÉTHODE MAGNÉTO-OPTIQUE}

\author{
Par Bernard HAGENE, \\ Laboratoire de Radioélectricité, Faculté des Sciences de Rennes.
}

\begin{abstract}
Résumé. - Si on perturbe par un procédé quelconque la température de spin d'un cristal paramagnétique, la détermination instantanée de la rotation Faraday optique permet de suivre la loi d'évolution de cette température et on dispose d'une méthode de mesure directe du temps de relaxation spin-réseau $T_{1}$. On décrit un appareillage qui permet d'enregistrer la rotation Faraday instantanée, dans des champs de 0 à 4000 gauss, et d'en déduire les temps de relaxation supérieurs à $10^{-4}$ seconde. On étudie les conditions optima permettant d'obtenir la meilleure sensibilité et le minimum de distorsion du signal. La perturbation de la température de spin est obtenue par addition rapide d'un champ magnétique supplémentaire créé par des bobines supraconductrices. Intérêt et limites de la méthode.
\end{abstract}

Abstract. - After disturbing the spin temperature of a paramagnetic crystal by any process, the instantaneous determination of the optical Faraday rotation allows one to follow the course of this temperature as a function of time, and we thus have a direct method for measuring the spin-lattice relaxation time $T_{1}$. We describe an apparatus for recording the Faraday rotation, in fields between 0 and 4000 gauss, from which the relaxation times higher than $10^{-4} \mathrm{~s}$ can be deduced. We study the best conditions for obtaining the highest sensitivity and minimum of distorsion. We disturb the spin temperature by sudden addition of a magnetic field produced by superconducting coils. Advantages and limitations of the method are discussed.

Introduction. - Kastler [1] a proposé d'appliquer l'effet Faraday optique souvent important présenté à basse température par certains sels paramagnétiques à la détection des transitions entre niveaux de spin, par exemple en résonance paramagnétique électro- nique, et des expériences ont été réalisées en particulier par Rieckhoff et Griffiths [2].

$\mathrm{Si}$ on perturbe par un procédé quelconque la température de spin d'un cristal, la détermination instantanée de la rotation Faraday $\beta$ permet de suivre 